Session No. 2221

\title{
Utilizing Educational Delivery Systems in a Mechanical Construction Course
}

\author{
Daryl L. Orth, Ph. D. \\ Purdue University \\ Department of Building Construction Management \\ West Lafayette, Indiana
}

In education, a delivery system is the organizational approach the instructor uses to deliver, organize, present, or communicate his/her instructional message or topic to the learners (Dick and Carey, 1996) ${ }^{1}$. The four most common delivery systems are non-projected media, projected visuals, audio, and video. All four delivery systems may be used in all three types of instruction. The three types of instruction are as follows:

- Instructor-led instruction is the traditional education approach where the student attends a classroom to receive face-to-face instruction from the instructor.

- The self-instructional delivery system, also known as packaged instruction, is where faceto-face instruction is combined with a multimedia kit allowing the student to learn at his or her own pace.

Distance education is where the instructor and student are separated by distance allowing for synchronous (live instruction and listening at a specific time) and asynchronous (non-live instruction) learning.

The purpose of an educational delivery system is to help the instructor arrange information and the environment that best facilitates learning (Heinich, Molenda, Russell, \& Smaldino, 1999) 2 In some instances, it is most appropriate to have the instructor deliver the entire instruction; while in other situations, a variety of instructional media may be employed. Instructional media are used in education to capture the students' attention, reinforce material, accommodate different learning styles, and provide opportunities for evaluation (Dick \& Carey, 1996) ${ }^{1}$. There is a wide variety of media that may be used in education such as non-projected media, projected visuals, audio, and computer networks/software (Heinich et al. 1999) ${ }^{2}$. To make the correct media choice, an instructor needs to know the advantages and disadvantages of the various types of media as well as the different learning styles. Presented here are brief descriptions of learning styles and instructional media and how they are employed in a mechanical construction class.

\section{Learning styles}

Before choosing a delivery system it is important that both the instructor and the students know how students best learn. A learning style is a preference for processing or acquiring new information. Every student will have a different leaning style so it is important to identify his or her own personal learning style. This allows for better retention of the information presented to them. Some students will learn better by listening; some by reading, and still others by viewing or performing a demonstration. 
There are four different learning styles: visual, aural, read/write, and kinesthetic. A visual learner comprehends best when information is presented visually such as in charts, diagrams, or pictures. Alternatively, an aural learner comprehends best by listening to the instructor, audio tapes, or group discussions. A read/write learner learns best by writing and reading text based items such as handouts and textbooks. However, a kinesthetic learner learns best by performing or manipulating hands on activities or demonstrations.

When teaching a subject, it should be explained or discussed using all four learning styles. For example, when discussing an item such as an air handler, the students have to read a short paper that discusses the function and components of an air handler. In the lecture portion of the class, the student are told about the function and components of an air handler using diagrams and pictures. In the lab portion of the class, students are actually shown an air handler and told about the function and components while the air handler is operating.

Non-projected media

Non-projected media are considered to be items such as real objects, models, mock-ups, field trips, printed materials, and chalkboards (Heinich et al. 1999) ${ }^{2}$.

Real Objects, Models, and Mock-Ups

While at first glance real objects, models, and mock-ups seem similar, they are in fact quite different. A model is a representation of a real object for either imitation or comparison, whether of larger or smaller scale as the real object (Heinich et al. 1999) ${ }^{2}$. A mock-up is no different than the mock-up panels created on a construction project for items such as exposed concrete aggregate and masonry brick veneer. A mock-up is a miniature model used in instruction to help highlight essential elements and to illustrate the basic operations of a real object (Heinich et al. 1999) ${ }^{2}$. All three of these media are excellent ways to show and explain complex construction details and the quality of workmanship or material that is expected.

Real objects, models, and mock-ups can be easily incorporated into a construction management curriculum. For example, this author was one of three instructors who were conducting a fourweek workshop introducing adult men and women to the different career opportunities in the field of construction. The participants were shown how to draft, estimate, and construct a 12foot by 16-foot outbuilding. First, the participants drafted the building; second, they estimated the material and labor cost for the building; and third, they actually constructed the building. The participants even had to shingle the roof, set windows and doors, install siding on the building, and provide plumbing for a kitchen sink and toilet. The three non-projected media items mentioned--real objects, models, and mock-ups--were all made available in this workshop: The tools they used such as power saws, hammers, nails, wood, levels, copper pipes, and blow torch were real objects. They were also shown a three-dimensional model of a house to help them visualize the different construction methods and details of the building. The model proved to be an excellent visual aid that also helped the workshop participants visualize what they were building. 
Real objects, models, and mock-ups were easily incorporated into the curriculum, and they were easily obtained. It seems that most construction students are visual or kinesthetic learners, so real objects, models and mock-ups are excellent instructional tools to help them to better understand the topic. Most classes require student projects accompanied with a presentation. Instructors could take advantage of this assignment by having the students create a model or mock-up as a presentation aid or tool. Such an assignment would help the students to think critically regarding their creation while helping them in the learning process. In addition, creating models or mock-ups would help the students to have a better presentation of their topics and make it easier for their classmates to understand their topics. It is also possible to obtain mock-ups from local contractors who are completing a construction project. Some contractors, as well as owners, are eager to help local schools and/or universities, and will donate the mockups that are no longer needed.

Field trips

A field trip is defined as an expedition outside the classroom that presents students with firsthand experiences of real, on-site processes, people, and objects. Field trips are an excellent way for students to encounter extraordinary topics or processes that may not be brought into the classroom for assessment (Heinich et al. 1999) ${ }^{2}$. In construction, field trips provide the students an opportunity to walk around the jobsite so they may experience the various activities of a construction project as well as talk to project supervisors. Plus, they actually see what construction is to determine if they truly wish to pursue a career in construction management. If they do want a construction management career, they are able to consider if they want to work in the residential or commercial aspect of construction.

To ensure that a field trip is valuable to the students, it should be appropriate to the class discussion. As an example, students have to purchase a set of construction blueprints for an oncampus building. The students are required to markup the drawings and identify all the HVAC and plumbing equipment and fixtures. To help the students understand and visualize the drawings, a field trip is taken to the on-campus building. Students are shown all the mechanical equipment that is labeled on the construction blueprints as well as the mechanical equipment that is not shown on the construction blueprints. This field trip helps the students to understand and visualize the construction blueprints. Additionally, a follow-up discussion of the field trip is conducted at the very next class. This gives student an additional opportunity to ask questions about the field trip. An excellent way to follow-up a field trip, would be to ask students to write a report that they are prepared to discuss in class regarding what they learned, what was most interesting, what they did not like, and any questions they have concerning the field trip.

Printed materials

Printed materials include textbooks, booklets, pamphlets, study guides, instructor/student manuals, worksheets, and other word-processed documents (Heinich et al. 1999) ${ }^{2}$. Printed materials have several advantages. They are readily available on a variety of topics and are userfriendly since they require no special effort to navigate. Additionally, printed materials may be adapted to facilitate several objectives. Printed materials are easy to transport and do not require special equipment or electricity, which also makes them user friendly. Probably the best

"Proceedings of the 2004 American Society for Engineering Education Annual Conference \& Exposition Copyright (C) 2004, American Society for Engineering Education”" 
advantage to printed materials is that they are reusable and are inexpensive to reproduce or purchase (Heinich et al. 1999) ${ }^{2}$.

Printed materials, however have several disadvantages. The reading level may be inappropriate or the reading topic may require prior knowledge. Most textbooks are written at a specific reading level, and a student with poor reading skills may not comprehend the material due to inadequate personal literacy skills. Textbooks also require a student to have some prior knowledge of the subject. Therefore, a student who lacks this prior knowledge will struggle to comprehend the material. Another disadvantage to printed materials is that they may be used in a passive method without understanding the message since printed material is not interactive. Perhaps the biggest disadvantage to printed materials such as textbooks is that they may dictate the curriculum rather than be used to support the curriculum (Heinich et al. 1999) ${ }^{2}$.

When trying to explain HVAC and plumbing systems, the author recommends that the blueprints of an actually construction project are obtained. Most project owners, architects, and engineers, are very willing in letting their construction drawings being used in an educational setting. A real life project is better to use than a set of drawings that are supplied with a textbook for several reasons. First, the students may actually visit the construction project to help them understand and visualize the process. Second, if a discrepancy is found in the drawings, the students may try to solve the problem and then find out how it was actually handled. Third, it gives the students a better sense of the quality of drawings and problems they will see when they are working in the industry.

\section{Chalkboards}

Dr. Carl Stafford stated "... that the chalkboard is probably the oldest and most familiar type of medium; however, it is probably the most misused medium in instructional delivery" (personal communication, June 18, 1999). Stafford continued by stating that the writing of some instructors is illegible on the chalkboard because they use several colors of chalk, have bad penmanship, or write notes in cursive. He also mentioned that it is easy to lose control of the audience because instructors will talk to the chalkboard and not to the audience. According to Stafford, the chalkboard may be used for a variety of reasons, but it is most effective when following these guidelines: The instructor should a) write, then turn facing the audience to talk, b) print using lower-case letters, c) write straight across, and use no more than five colors of chalk, and d) use templates to draw shapes. Some additional information Carl Stafford provided is that the larger the room, the larger the print needs to be. He recommended that the letters should be 1 inch tall for every 10 feet of the room size.

Projected Visuals

Projected visuals are defined as media formats in which still images are projected onto a screen $\left(\right.$ Heinich et al. 1999) ${ }^{2}$. Items such as overhead transparencies and PowerPoint slides are projected visuals.

Overhead Transparencies 
Overhead transparencies seem to be one of the most versatile and popular presentation mediums used today. Overhead transparencies encourage the students to participate as well as provide a relaxed, controlled atmosphere that permits students to learn (Kupsh, 1999) ${ }^{3}$. One of the principal advantages to using overhead transparencies is that they allow the instructor to face the audience, making eye contact that is necessary for effective two-way communication (Kupsh, 1999) ${ }^{3}$. When using overhead transparencies, the instructor needs to make sure that the overhead projector is focused, and that the room is dimly lit by dimming the classroom lights and closing any window blinds. With a dimly lit room, students should be able to see the overheads properly. Be careful not to make the room too dark; otherwise, the students could fall asleep, and more important, it would be difficult for them to take notes.

Information on the overhead transparency should be exposed point by point, preventing the students from reading ahead or jumping to conclusions. When exposing each point, the instructor is encouraged to place his or her cover-up sheet underneath the transparency, so that he or she may view the next points of discussion while the students see only the current point. A quick guide to tell if the print on the overhead transparency is large enough is to place the transparency on the floor. Stand up directly above the overhead transparency; if the text on the overhead transparency may be easily read, then the text is large enough.

When finished with overhead projector, it should be turned off to maintain student focus. All major points of the overhead should be clarified by highlighting, underlining, or circling to prevent confusion among the students (Kupsh, 1999) ${ }^{3}$. When writing on overhead transparencies, the instructor needs to print. If a permanent marker is mistakenly used instead of an overhead projector marker on an overhead transparency, the permanent marker may be easily removed by writing over it with an overhead projector marker, and then wiping with a cloth (J. D. Russell, personal communication, June 18, 1999). If the overhead projector has a roller for the transparency film, it is recommended that the overhead transparency be placed under it while discussion is taking place. This way when the discussion is finished, the overhead transparency is still clean.

\section{Microsoft PowerPoint}

Microsoft PowerPoint is an excellent way to show clear, full-colored, three-dimensional still pictures with detailed graphics of mechanical equipment. The main advantage to using PowerPoint is that slides may be easily created, edited, and duplicated. Microsoft PowerPoint also allows the easy incorporation of digital photography. Pictures may be taken from field trips or other construction jobsites with a digital camera and then incorporated into PowerPoint. Once the picture is in PowerPoint, text and other animation may be added to highlight key points. The main disadvantage to using PowerPoint slides is that they involve little or no audience participation. One way to combat this problem is to get the students involved by asking them a question or to explain the slide or picture. Instructors who use PowerPoint considerably during their instruction need to make a conscious effort to invoke student participation or incorporate other media formats that encourage student participation (Kupsh, 1999) ${ }^{3}$.

When creating PowerPoint slides it is recommended to use a light background with dark text or graphics for a couple reasons. The first reason is that the light background and dark text will

\footnotetext{
"Proceedings of the 2004 American Society for Engineering Education Annual Conference \& Exposition Copyright (C) 2004, American Society for Engineering Education"
} 
provide enough contrast so the slides may be easily seen. The second reason is that the room will not have to be as dark to view the pictures. A darkened room for an extensive period of time may make the students drowsy. When using PowerPoint presentation, the instructor needs to remember to face and talk to the audience at all times. It is also recommended to end the presentation with a blank slide. This alerts the instructor and the students that it is the end of the presentation.

When using overhead transparencies and Microsoft PowerPoint, it is important that the 36 rule be followed. Meaning no more than 6 lines, with 6 words per line, should appear on a transparency or slide (J. D. Russell, personal communication, June 18, 1999).

\section{Audio}

Audio media such as the instructor's voice is an important medium that should not be overlooked since it is estimated that college students will spend approximately 90 percent of their time in class listening to lectures and seminar discussions (Heinich et al. 1999) ${ }^{2}$.

\section{Voice}

An instructor's voice plays an important role in receiving and retaining student interest (Kupsh, $1999)^{3}$. One of the biggest problems with straight lectures is an instructor who speaks with a monotone voice which causes students to suffer from auditory fatigue. Auditory fatigue occurs when a listener becomes unaware or loses concentration due to a repetitious monotonous sound. The instructor needs to vary his or her tone of voice as well as change the pace of the instruction to help maintain the student's attention. Another important point for the instructor to remember is to speak slowly and clearly enunciating each word correctly so that everyone may easily hear the instruction. Students will be lost if they have to struggle to hear the instructor's voice (Kupsh, 1999) ${ }^{3}$. Additionally, an instructor needs to be enthusiastic and interested in the topic that is being taught. If the instructor is not enthusiastic or does not care about the instructional topic, the students will definitely not be interested or excited. It is beneficial to incorporate some humor into the lecture by telling a story or joke that is related to the topic being discussed to help relay the message. Telling a true story will provide the instructor with some creditability (Kupsh, 1999) ${ }^{3}$.

\section{Video}

The primary meaning of video is the displaying of images on a television-type screen (Heinich et al. 1999) ${ }^{2}$. Video is adaptive for almost every situation, and is one of the most effective delivery systems when the instruction requires real-life action. Video clips may be used with computer graphics to create multimedia presentations that include text, graphics, animation, and motion video (Kupsh, 1999) ${ }^{3}$.

Videos are a great aid for instruction or training, and make remembering details much easier since the details may be visualized. Videos are so adaptable that they may be used for a variety of purposes such as educational demonstrations, problem solving, general information, and/or for critiquing construction materials and methods. Video presentations offer full-color motion and 
may be more entertaining. In addition, video presentations look professional since today's technological developments are making the recording, capturing, duplicating, viewing, editing, and printing of video images easier, more practical, and more cost effective (Kupsh, 1999) ${ }^{3}$. One of the biggest advantages to using video is that videos allow us the opportunity to increase or decrease the amount of time needed to study an incident or method.

One of the disadvantages of using video presentations is that they may be impersonal and discourage audience participation since little presenter or viewer involvement is required. Furthermore, viewing videos is a passive activity and may allow the audience to lose concentration and focus (Kupsh, 1999) ${ }^{3}$. Another possible disadvantage of using videos as a teaching aid is that if the video is too entertaining, students may overlook the educational information contained in the video.

Videos on different mechanical construction topics may be obtained from several construction professional organization such as the Mechanical Contractors of Association of America, Plumbing Heating, Cooling Contractors, or American Society for Heating, Refrigeration, and Air Condition Engineers. When showing a video, ask the students to look for a specific idea or activity in the video before viewing. Then immediately after the video presentation, ask questions specifically about the material that was shown in the video. A list of questions may also be handed to the students that should be answered as the video is showing.

\section{Conclusion}

The delivery system by which the instructional unit is delivered to students may have a significant impact on the student's learning experience. Each delivery system may be used in all three types of instruction because each has attributes that make it more or less appropriate depending on the situation. When selecting a delivery system, be sure to first learn the students' individual learning styles, and then create the course objectives because these criteria will determine the medium.

In some instances, it is going to be appropriate to have the instructor deliver the entire instruction, while in other situations, a variety of media may be employed. The different delivery systems such as non-projected media, projected visuals, audio, and video are used in education to capture the students' attention and to meet the needs of various learning styles. The delivery system should help to reinforce the instructional topic and provide a way to evaluate student performance. Incorporating a variety of delivery systems into the instruction should accommodate for the various learning styles. There is a wide variety of delivery systems that may be used in education and the delivery systems mentioned in this paper are only a survey of what is available. To make the correct media choice, an instructor needs to know the advantages and disadvantages of the various types of delivery systems.

References

1. Dick, W. \& Carey, L. (1996). The systematic design of instruction $\left(4^{\text {th }}\right.$ ed.). New York: Longman. 
2. Heinich, R., Molenda, M., Russell, J. D., \& Smaldino, S. E. (1999). Instructional media and technologies for learning $\left(6^{\text {th }}\right.$ ed.). Upper Saddle River, New Jersey: Prentice-Hall, Inc.

3. Kupsh, J. (1999). Presenter's guide. Presenter's Information Center [On-line]. Available: www.thepic.com/guide/index.html.

Biographical Information

DR. DARYL L. ORTH, PH.D. is an assistant professor and coordinator of the mechanical construction management program at Purdue University in the Department of Building Construction Management (BCM). Dr. Orth started teaching in the BCM Department in the Fall of 2000. He is responsible for teaching all their mechanical construction classes. 